\title{
اثر همارسة النشاط الرياضي في سمك بطانة الرحم وبعض التغيرات المصاحبة للدورة الشهرية
}

\section{م.د. وسن سعيد رشيد م.د. نشوان إبراهيم عبد الله}

\section{هلفـص البحسث}

هدف البحث إلى الكثف عن اثر ممارسة النشاط الرياضي في سمك بطانة الرحم ويعض التغيرات المصاحبة للاورة الثهرية،ولبلوغ ذلك فقد تم اختيار ( (Y) فتاة بالطريقة العمدية تم تقسيمهم إلى مجموعتين

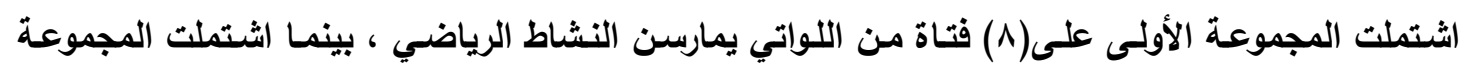

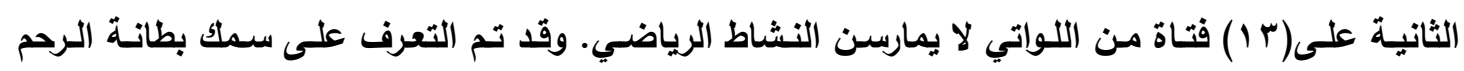
باستخدام جهاز الأمواج فوق الصوتية (جهاز السونار) في حين تم التعرف على التغيرات المصاحبة للاورة

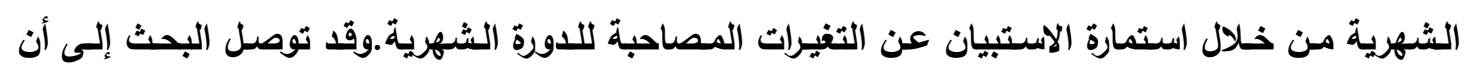
ممارسة النشاط الرياضي تؤدي إلى التقليل من سمك بطانـة الرحم وكذلك تؤدي إلى زيـادة النسبة المئويـة

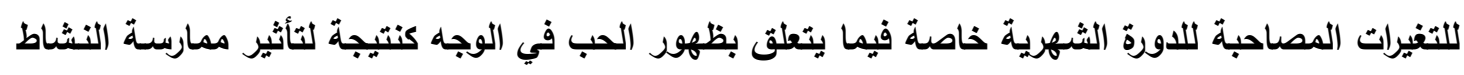

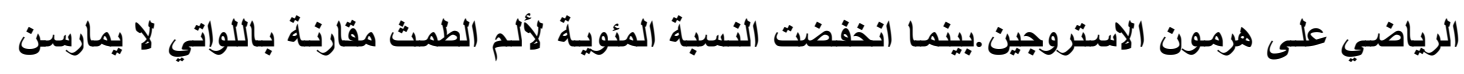

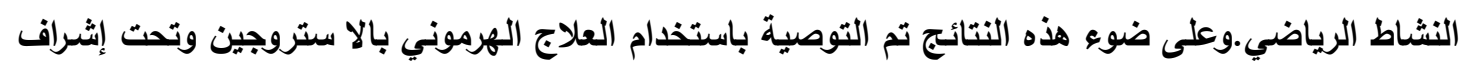
طبي وذلك للتقليل آو لتلافي قلة سمك بطانة الرحم وظهور الحب في الوجه لاى الممارسات للنشاط الرياضي. 


\section{Abstract \\ Effect of physical activities on endometrium thickness and some associated variables with menstrual cycle}

In recent years ,the research attention has been focused on woman,s exercise responses and the effect of exercise on and the affect on exercise of the menstrual cycle.The purpose of this study was to investigate the effect of physical activity on endometrium thickness and some associated variables with menstrual cycle. Twenty one females females participated in this study divided in two groups ,first group included $(\mathrm{n}=8)$ athletes females $(23.4 \pm 2.7 \mathrm{yrs}, 55.22 \pm 7.003 \mathrm{~kg}, 1.60 \pm 0.11)$, and the second group included $(\mathrm{n}=13)$ non-athletes females $(22.7 \pm 3.3 \mathrm{yrs}, 57.06 \pm 10.2 \mathrm{~kg}$, $1.56 \pm 0.14 \mathrm{~m})$. endometrium thickness had been measured by ultrasound method, while the associated variables with menstrual cycle was obtained by questionnaire. The questionnaire included questions about headache, abdomen pain and back pain, pimples in the face region, breast distension, and menses pain. There was significant decrease in percentage for endometrium thickness in athletes females $(\% 0.72)$ in comparison with the non-athletes females $(\% 0.21)$, and also there was significant increase in the percentage for the associated variables with menstrual cycle in athletes females especially pimples in the face region( $\% 0.28)$, while we was found a significant increase in the percentage for menses pain in non- athletes females $(\% 0.82)$ in comparison with athletes females $(\% 0.24)$.This study concluded that active females show decrease in percentage for endometrium thickness and increase in associated variables appearance with menstrual cycle (pimples in the face region) and decrease menses pain in comparison with non-athletes females. The percentage decrease in endometrium thickness, and percentage increase in pimples in the face region athletes females attribute to the decrease in the level for Estrogen hormone as a result of the effect of physical activity, also the hormone decrease lead to increase in androgen hormone activity that causes to appearance the pimples in the face region. Thus, this result suggest using the hormone therapy with estrogen under physician supervision in order to reduce the Effect of physical activities on endometrium thickness and some associated variables with menstrual cycle. 


\section{البـاب الأول}

|-التعريف بالبمث

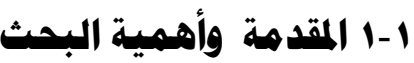

لقد استأثرت فكرة ممارسـة المرأة للنشاط الرياضـي اهتمـام الباحثين والمختصين في مجال فسلجة

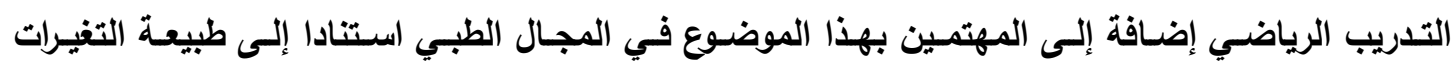

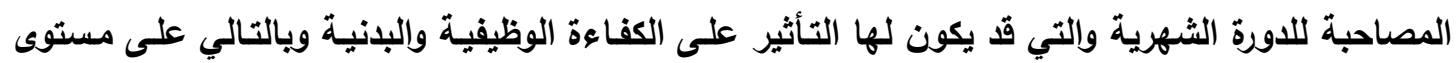

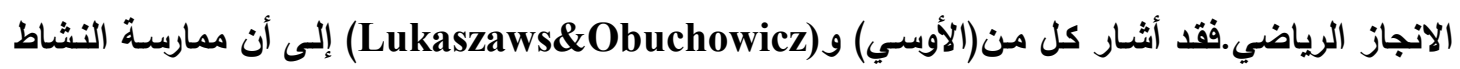

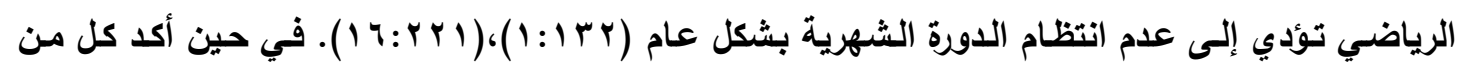

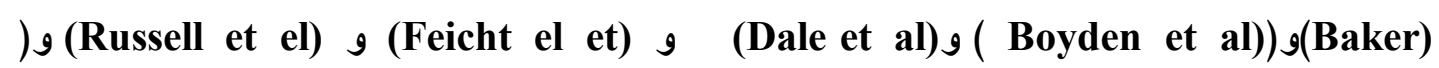

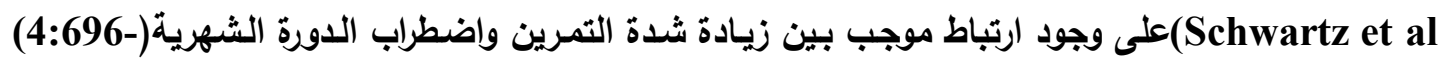

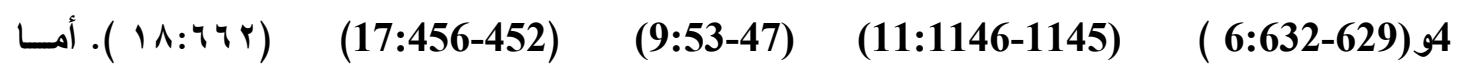
(Fox\&Mahtews) و (Edwin) و) (George\&Elwood)

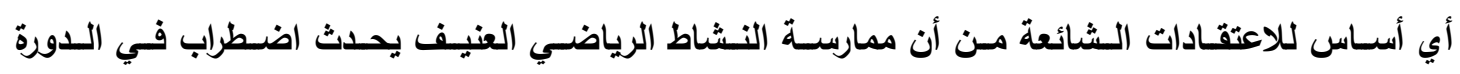

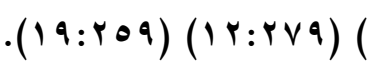
10:53-47

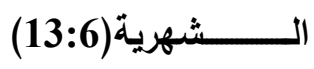

مما تقدم نلحظ أن معظم الاراسات تركزت حول تأثير ممارسة النشاط الرياضي في اضطراب الدورة الشهرية

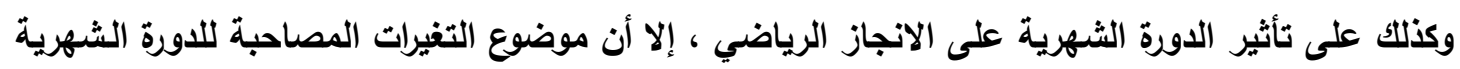

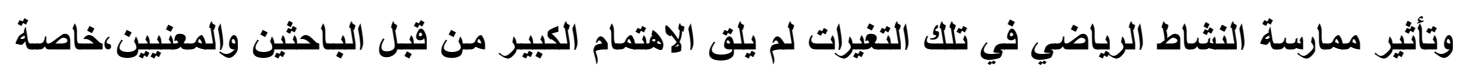

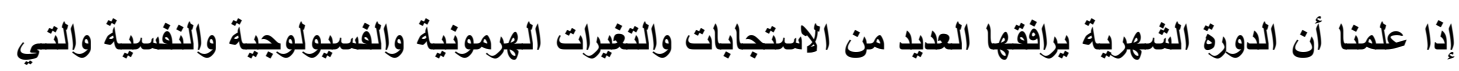

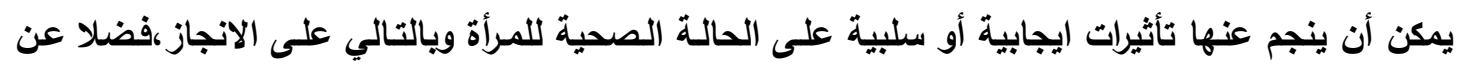

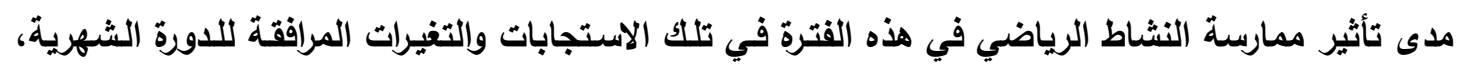

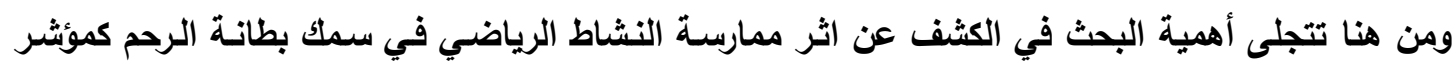

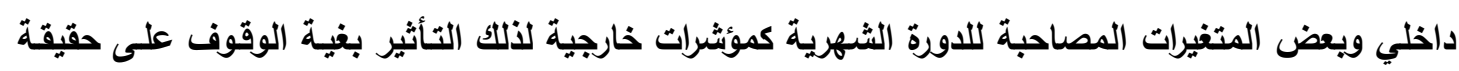

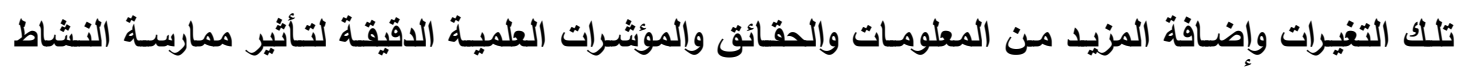

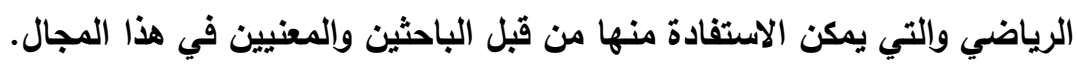

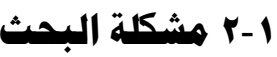

تحددت مشكلة البحث في النقاط الآتية :

يرافق الدورة الشهرية العديد من الاستجابات والتغيرات الفسيولوجية والهورمونية والنفسية والتي يكون لهاتيات تأثيرات كثيرة سواء على المستوى الصحي للمرأة أو الانجاز الرياضي،والتساؤل المطروح هو هل أن ممارسية

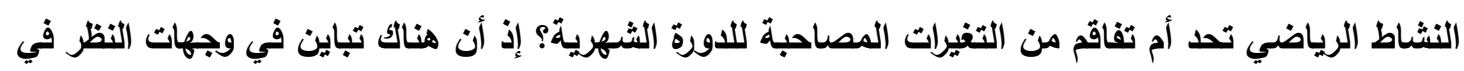
ما يخص تأثير ممارسة النشاط الرياضي في التغيرات المصاحبة للاورة الشهرية (الداخلية والخارجية) للى

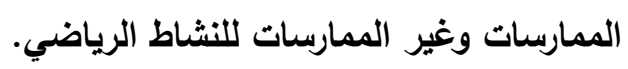
تركيز معظم الدراسات والأبحاث على دراسة تأثير الدورة الثهرية في الانجاز أو على تأثثر الجهد البدني أو أو الثان

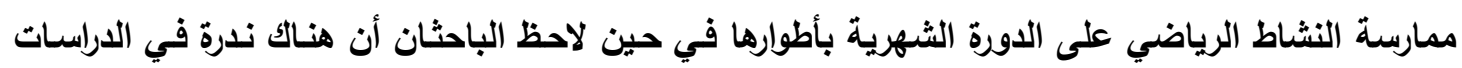


والأبحاث التي تناولت تأثير ممارسة النشاط الرياضي في سمك بطانة الرحم ويعض التغيرات المصاحبة للاورة

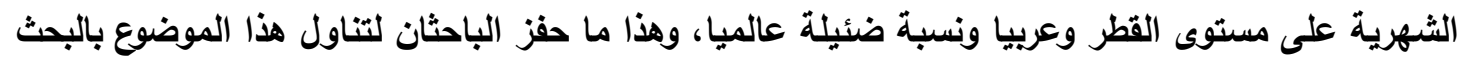
والدراسة.

r- r r أهد|ف البهث

1. الكثف عن اثر ممارسة النشاط الرياضي في سمك بطانة الرحم والتغيرات المصاحبة للاورة الثهرية لاى الممارسات للنشاط الرياضي. r. الكشف عن اثر ممارسة النشاط الرياضي في سمك بطانة الرحم والتغيرات المصاحبة للاورة الثهرية لاى غير الممارسات للنشاط الرياضي. r. المقارنـة بين تأثير الممارسـة وعدم الممارسـة للنشاط الرياضـي في سـك بطانـة الرحم والتغيرات المصاحبة للاورة الشهرية لاى الممارسات وغير الممارسات للنشاط الرياضي.

1 - ه فروض البمث

ا. وجود فرق ذات دلالة معنويـة بين الممارسـات للنشاط الرياضسي وغير الممارسـات في سمك بطانة

$$
\text { الرحم والتغيرات المصاحبة للاورة الشهرية. }
$$

r. اختلاف النسبة المئويـة للتغيرات في سمك بطانة الرحم والتغيرات المصاحبة للدورة الشهرية بين الممارسات وغير الممارسات للنشاط الرياضي.

\section{1 -0 هجالات البحث}

1 -1-1 المجال البشري : من الممارسات للنشاط الرياضي ومن غير الممارسات للنشاط الرياضي .

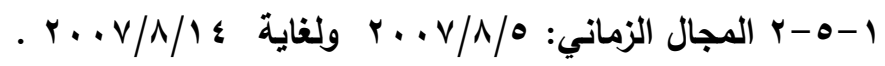

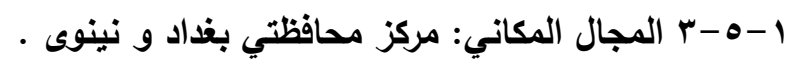
البــاب الثانسي لودي

r - الدراسات النظرية The Menstrual Cycle الدورة الشهرية تعرفها الأوسي بأنها سلسلة من التغيرات الوظيفية في بطانـة الرحم التي تحدث كل(28) يوم في

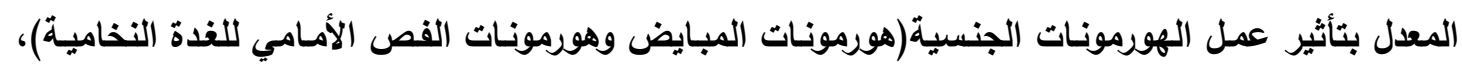

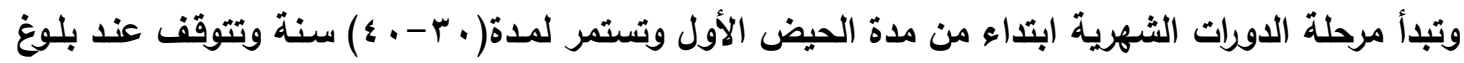

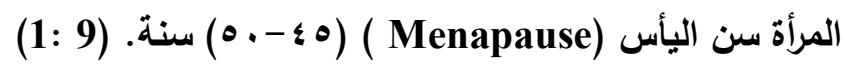

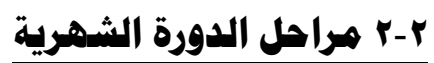

\section{Proliferative Phase المرحلة الأولى: هرحلة التعمير أو البناء}

وهي المرحلة التي تمتد من اليوم الخامس بعد نزول الحيض مباشرة إلى اليوم الرابع عشر من

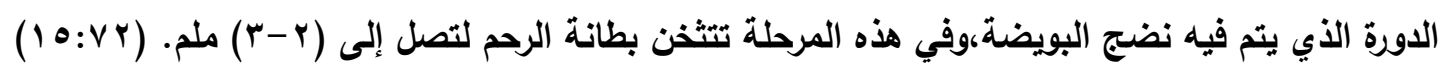




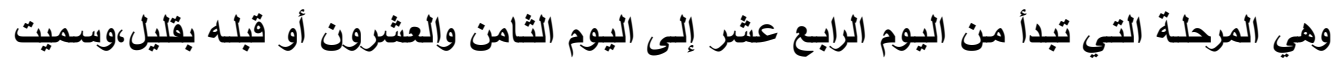
بالمرحلة الإفرازية نتيجة لنشاط الغد الإفرازي ، ويكون النسيج ألطلائي المبطن للرحم قد بلغ أقصى سمك له.

\section{Destructive Phase المرحلة الثالثة : هرحلة التحطيم(هرحلة الهيض)}

وهي المرطلة التي ينزف فيها الام وتبدأ في نهاية المرحلة السابقة وتستمر (ع -ه) أيام،وخلال

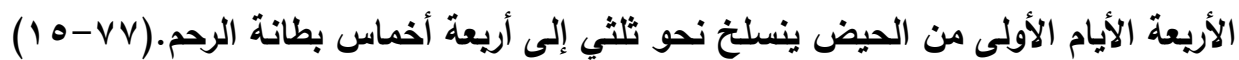

\section{البــاب الثالـث}

r- ب إجراءات البحث

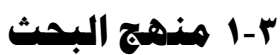

تم استخدام المنهج الوصفي لملائمته لطبيعة البحث.

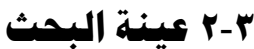

اشتملت عينة البحث على (Y ( ا برأة قسمن إلى مجموعتين تكونت المجموعة الأولى من(^) نساء

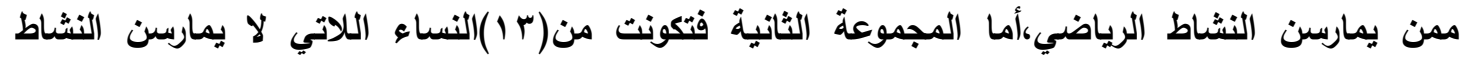
الرياضي. والجدول (1) يبين بعض مواصفات عينة البحث.

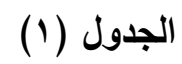

يبين بعض المعالم الإحصائية لبعض المعلومات عن عينة البحث

\begin{tabular}{|c|c|c|c|c|}
\hline (سنة) & الطول & (كف) & الإحصائية & المجموعة \\
\hline צ. & 1.7 & YY.OD & س & \multirow{2}{*}{ الممارسات للنثاط } \\
\hline V.r &. .11 & $v . . r$ & $\varepsilon \pm$ & \\
\hline rY.V & 1.07 & OV.. T & سَ & \multirow{2}{*}{ غلنشاط الرياضي } \\
\hline$r . r$ &. $.1 V$ & $1 . . r$ & $\varepsilon \pm$ & \\
\hline
\end{tabular}

r-r الأجهزة والأدوات المستخدهة في البحث

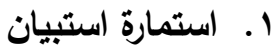

r. جهاز سونار(Ultra Sound) نوع) (Siemens, Sonline-SX) .

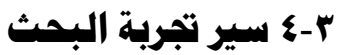

بغية التحديد اللقيق لتأثير ممارسة النشاط الرياضي في سمك بطانة الرحم والتغيرات المصاحبة

للاورة الثهرية والوقوف على مؤثراتها العلمية تضمنت تجرية البحث الإجراءات الآتية :

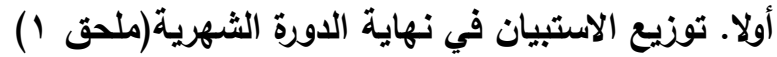

ثنانيا : فحص بطانة الرحم باستخدام جهاز السونار (Ultra Sound). 
تم إجراء فحص بطانة الرحم " لكل عينة ولكلتا المجموعتين بعد مرور (ب ا - ـ ا ) يومـا من موعد

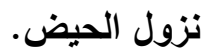

\section{ب-r الوسائل الإحصائية}

تم استخدم الوسائل الإحصائية الآتية:

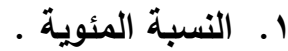

r . الوسط الحسابي •

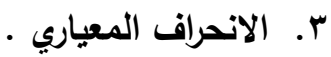

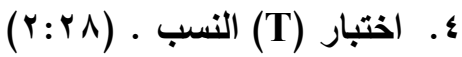

\section{البـاب الرابــع}

؟- عرض ومناقشة النتائج

؟-ا عرض وهناقشة نتائج سمك بطانة الرحم

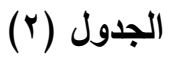

يبين المعالم الإحصائية لسمك بطانة الرحم بين الممارسات وغير الممارسات للنشاط الرياضي

\begin{tabular}{|c|c|c|c|}
\hline \multirow{2}{*}{ قالنسبة (تئة المئوية } & \multicolumn{2}{|c|}{ سمك بطانة الرحم } & \multirow{2}{*}{ المجموعة } \\
\hline & قليلة السمك \% & طبيعية \% & \\
\hline \multirow{2}{*}{$*$ *.r। rq } &.$v r$ & $99 . Y \wedge$ & الممارسات للنشاط الرياضي \\
\hline &.$r_{1}$ & $99 . \vee 9$ & غير الممارسات للنشاط الرياضي \\
\hline
\end{tabular}

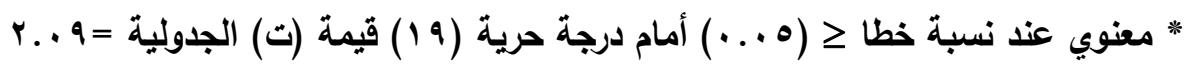

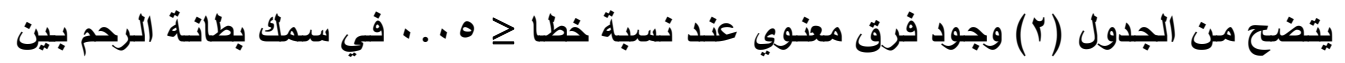

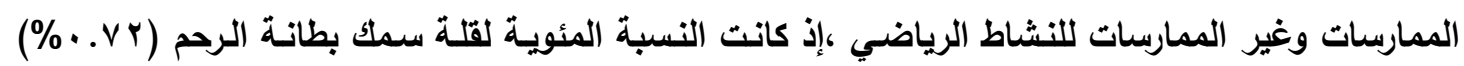

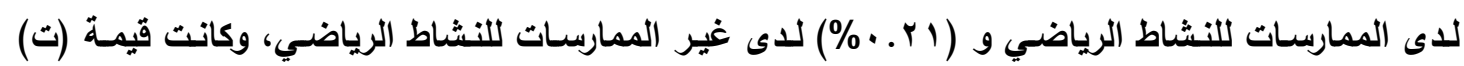

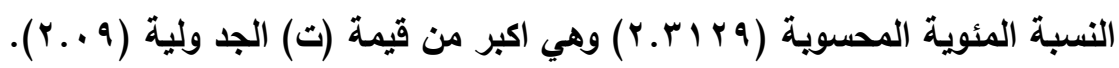

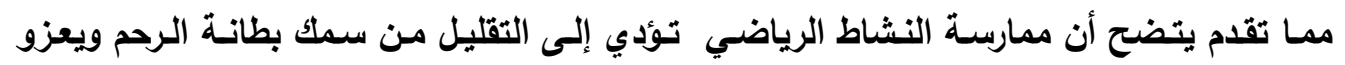

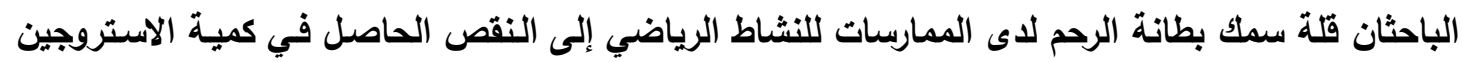
،إذ يؤدي نقصه إلى نقص سمك بطانة الرحم، ويعود سبب هذا النقص إلى تأثير النشاط البدني وان ما يؤكد et $\underline{\text { al) }}$

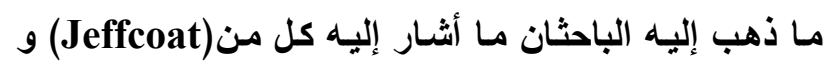
(Boyden وـ (Baker et

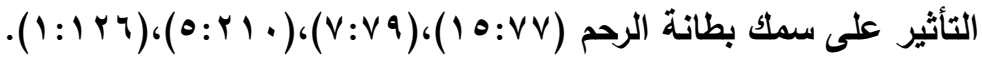

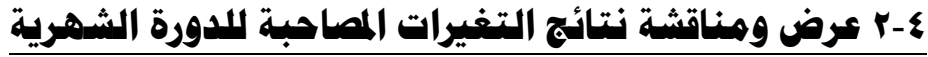

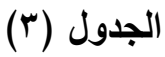


يبين المعالم الإحصائية للتغيرات المصاحبة للاورة الشهرية بين الممارسات وغير الممارسات للنشاط الرياضي

\begin{tabular}{|c|c|c|c|}
\hline النسبة المئوية (ت) & \multicolumn{2}{|c|}{ النسبة المئوية لوجود التغيرات المصاحبة للاورة } & المتغيرات \\
\hline \multirow{2}{*}{$*$ *.11VI } & $\cdot v_{1}$ & ممارسات & \multirow{2}{*}{ البثور والحب في الوجه } \\
\hline &.$r \wedge$ & غير ممارسات & \\
\hline \multirow{2}{*}{. } & $\cdot . \leqslant V$ & ممارسات & \multirow{2}{*}{ الصداع } \\
\hline & .0 & غير ممارسات & \\
\hline \multirow{2}{*}{ I.rAmq } &.$V r$ & ممارسات & \multirow{2}{*}{ الم البطن والظهر } \\
\hline & 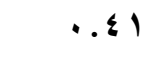 & غير ممارسات & \\
\hline \multirow{2}{*}{1.0790} & $\because V V$ & ممارسات & \multirow{2}{*}{ انتفاخ الثدي } \\
\hline & $\cdot . \leqslant r$ & غير ممارسات & \\
\hline \multirow{2}{*}{ *Y.7rצะ } &.$r \varepsilon$ & ممارسات & \multirow{2}{*}{ الم الطمث(مغص حاد) } \\
\hline &.$\Delta r$ & غير ممارسات & \\
\hline
\end{tabular}

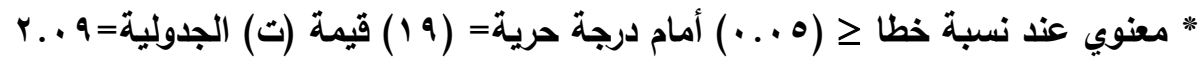
يتبين من الجدول (Y) ما يأتي :

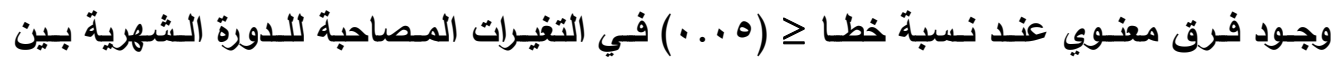
الممارسات وغير الممارسات للنشاط الرياضي في متغير ظهور البثور والحب في الوجه ولصالح الممارسـات

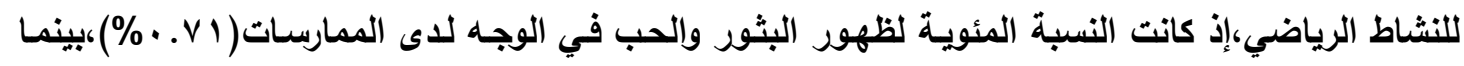

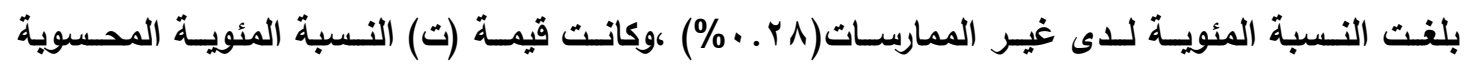

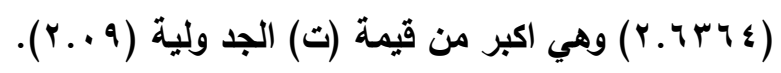

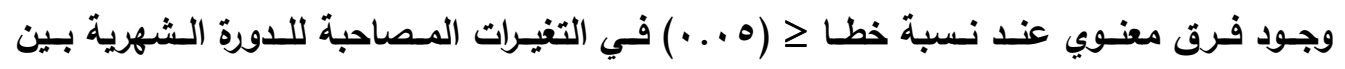

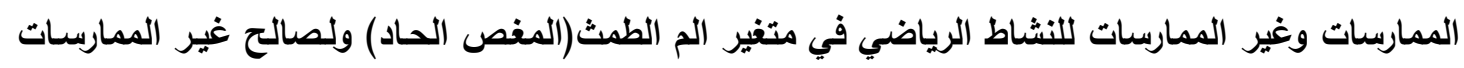

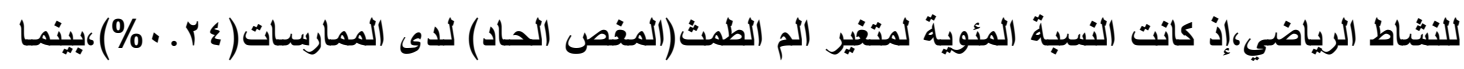

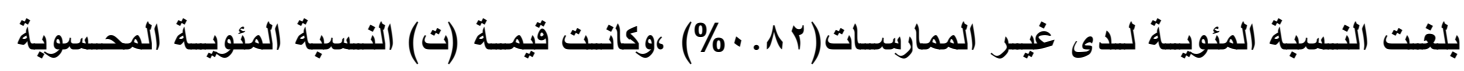

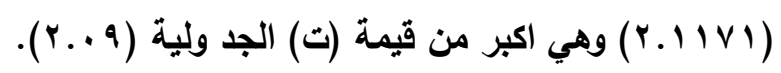

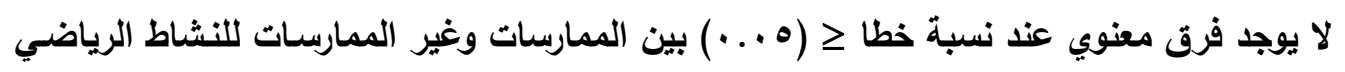

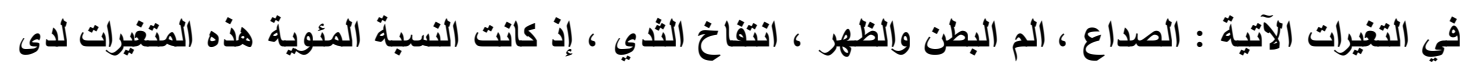

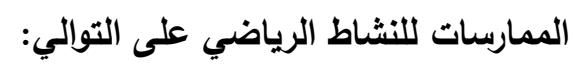

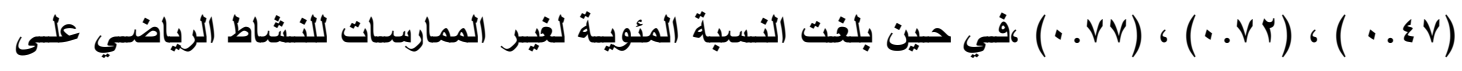

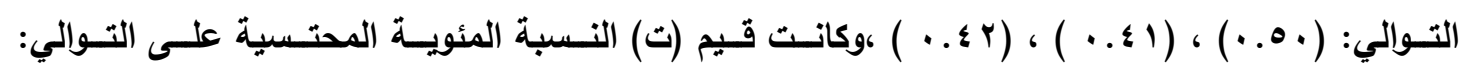

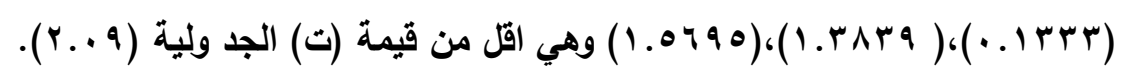
مما تقدم يتبين ما يأتي : 
إن ممارسـة النشاط الرياضـي تؤدي إلى ظهور البثور والحب في الوجـه كأحد التغيرات المصاحبة للنشاط الرياضي،بينما تؤدي عدم ممارسة النشاط الرياضسي إلى زيادة الم الطمث (المغص الحساد) ، ويعزو الباحثان

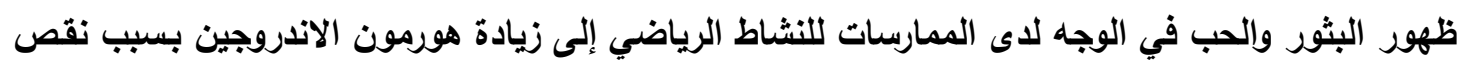
الاستروجين من جراء ممارسة النشاط الرياضي.ويرى الباحثان أن التفسير الذي ذكره (Hoeger\&Hoeger)

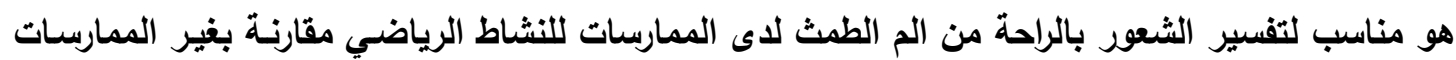
للنشاط الرياضي إذ يشيران إلى أن سبب ذلك يعود إلى تحسن الدورة الدموية للرحم ،إضافة إلى زيادة مستوى لهن

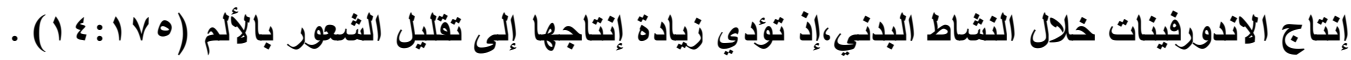




\section{البـاب الخاهـسس}

0- الاستنتتاجات والتوصيات

0-1 - الاستنتاجات

ا ـ تؤدي ممارسة النشاط الرياضي إلى التقليل من سمك بطانة الرحم وزيادة ظهور البثور والحب في

الوجه.

r . تؤدي عدم ممارسة النشاط الرياضي إلى زيادة الثعور بألم الطمث(المغص الحاد).

r. لم تظهر ممارسة وعدم ممارسة النشاط الرياضي أي تباين في التغيرات المصاحبة للاورة الشهرية

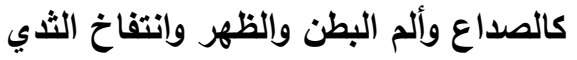

r-o r r التوصيات

ا. لتقليل وتلافي قلـة سـكك بطانـة الرحم وظهور البثور والحب في الوجـه يفضل استخدام العـلاج

الهورموني بالاستروجين وتحت إثراف طبي ·

ץ. إجراء دراسات أخرى تتناول تأثير ممارسة النشاط الرياضي للأجزاء الأخرى للجهاز التناسلي الأنثوي

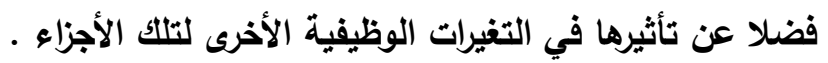

\section{المصسادر العربيـيسة والأجنبيسيسة}

ه الأوسي،وسن سعيد رشيد (999 199) : تأثير الجها البذني في البلوغ واضطرابات الدورة الشهرية عند

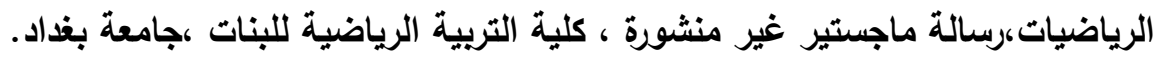

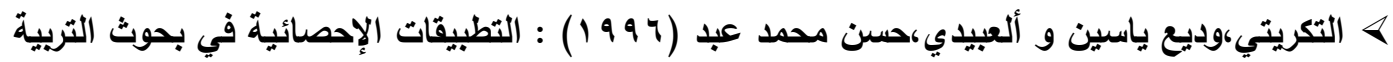

الرياضية،دار الكتب للطباعة والنشر، جامعة الموصل.

Arlene E., and Howard E (1979): A live Well . McGraw-Hill Poblisher .

Baker E.R (1981): Menstrual dysfunction and hormonal status in athletic woman: A review fertile. Sterile .(36).

$>$ Baker E.R., and others (1982): Amenorrhea associated with running mileage and age. Physician and Sports Medicine, Vol (10),No(10),McGraw-Hill Publisher, U.S.A

$>$ Boyden T.W., and others (1983): Sex steroids and endurance running in women. Fertile. Sterile (39).

$>$ Boyden T.W., and others (1984): Training caused significant decreases in plasma estradiol . Physician and Sports Medicine, Vol (12), No(1). McGrawHill Publisher, U.S.A

$>$ Christine L.W. (1985): Women, Sport of performance. Human Kinetics Publishers.

$>$ Dale E., and others (1979): Menstrual dysfunction in distance runner. Obstet. Gynecol (54).

Edwin D., and others (1980): Menstrual dysfunction . Obstet. Gynecol (56).

$>$ Fiecht C.B., and others (1978): Secondary amenorrhea in athletes. Lancet (2).

$>$ Fox E.L., and Mathews D.K. (1981) :The physiological basis of physical education and athletics $.3^{\text {rd }}$ Edition. Saunders College Publishing . 
$>$ George J.H., and Elwood G.D. (1975): Values of physical activity. $3^{\text {rd }}$ Edition. WM.G.Brown Publishers.

Hoeger W.K., and Hoeger S.H (1990): Fitness and Wellness. Morton publishing Company. U.S.A .

Jeffcoat S. (1987): Principles of Gynecology . Butterworth World Student Publishers. London.

Lukaszewska J.H., and Obuchowicz F.B. (1985): Serum and urinary steroids in women athletes. J. Sports Med. Vol (25). No(4).

Russle J.B., and others (1984): The relationship of exercise to an voluntary cycle in female athletes : Hormonal and physical characteristics. Obstet. Gynecol.

$>$ Schwartz B., and others (1981): Exercise associated amenorrhea. Am. J. Obstet. Gynecol. (141).

$>$ Struass H.R. (1979): Sport medicine . WB Saunders Company. 


\section{الملاحـــ \\ (1) ملـــ \\ استهارة استبيان}

تضمنت استمارة الاستبيان التساولات الآتية :

1. هل تعانين من تغيرات جلدية خلال الدورة الثهرية؟ كظهور الحب في الوجه أو ظهور بثور أو تغير

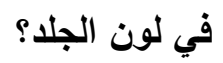

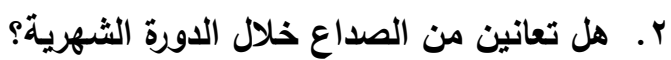

r. هل هل تثعرين بالألم في منطقة البطن والظهر خلال الدورة الثهرية؟

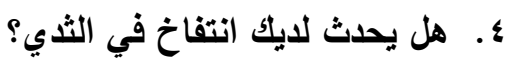

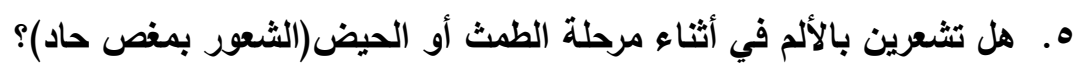

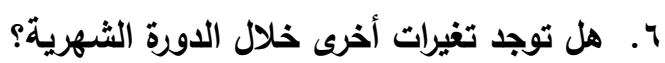

